\title{
Person-centred medicines optimisation policy in England: an agenda for research on polypharmacy
}

\author{
Janet Heaton ${ }^{1}$, Nicky Britten ${ }^{2}$, Janet Krska ${ }^{3}$ and Joanne Reeve ${ }^{4}$ \\ ${ }^{1}$ Research Fellow, Institute of Health Research, University of Exeter Medical School, Exeter, Devon, UK \\ ${ }^{2}$ Professor, Institute of Health Research, University of Exeter Medical School, Exeter, Devon, UK \\ ${ }^{3}$ Professor, Medway School of Pharmacy, Universities of Greenwich and Kent, Chatham Maritime, Kent, UK \\ ${ }^{4}$ Associate Clinical Professor, Warwick Primary Care, Division of Health Sciences, Warwick Medical School, University \\ of Warwick, Coventry, Warwickshire, UK
}

\begin{abstract}
Aim: To examine how patient perspectives and person-centred care values have been represented in documents on medicines optimisation policy in England. Background: There has been growing support in England for a policy of medicines optimisation as a response to the rise of problematic polypharmacy. Conceptually, medicines optimisation differs from the medicines management model of prescribing in being based around the patient rather than processes and systems. This critical examination of current official and independent policy documents questions how central the patient is in them and whether relevant evidence has been utilised in their development. Methods: A documentary analysis of reports on medicines optimisation published by the Royal Pharmaceutical Society (RPS), The King's Fund and National Institute for Health and Social Care Excellence since 2013. The analysis draws on a non-systematic review of research on patient experiences of using medicines. Findings: The reports varied in their inclusion of patient perspectives and person-centred care values, and in the extent to which they drew on evidence from research on patients' experiences of polypharmacy and medicines use. In the RPS report, medicines optimisation is represented as being a 'step change' from medicines management, in contrast to the other documents which suggest that it is facilitated by the systems and processes that comprise the latter model. Only The King's Fund report considered evidence from qualitative studies of people's use of medicines. However, these studies are not without their limitations.

We suggest five ways in which researchers could improve this evidence base and so inform the development of future policy: by facilitating reviews of existing research; conducting studies of patient experiences of polypharmacy and multimorbidity; evaluating medicines optimisation interventions; making better use of relevant theories, concepts and tools; and improving patient and public involvement in research and in guideline development.
\end{abstract}

Key words: guidelines; medicines optimisation; multimorbidity; patient-centred prescribing; person-centred care; polypharmacy

Received 27 November 2015; revised 14 April 2016; accepted 22 May 2016;

first published online 16 June 2016

Correspondence to: Dr Janet Heaton, Research Fellow, PenCLAHRC, University of Exeter Medical School, South Cloisters (Rm 1.36), St Luke's Campus, Exeter EX1 2LU, Devon, UK. Email: j.heaton@exeter.ac.uk

\section{Introduction}

There have been some important developments in policy on medicines optimisation in England in recent years. In 2013, the Royal Pharmaceutical Society (RPS) (Picton and Wright, 2013) and

(C) Cambridge University Press 2016 
The King's Fund (Duerden et al., 2013) separately published independent reports describing the rise of polypharmacy and promoting strategies for optimising the growing use of multiple medicines by individual patients. These reports were followed by the production of official guidelines on medicines optimisation by the National Institute for Health and Social Care Excellence (NICE, 2015a). In this paper, we critically examine the ways in which patient perspectives have been incorporated in these influential independent reports and official guidelines. We also highlight gaps in research on people's experiences of polypharmacy that need to be addressed in order to inform the future development of more inherently person-centred medicines optimisation policy and practice in the National Health Service (NHS).

\section{Methods}

We carried out a non-systematic review of the English-language research literature relating to patient experiences of polypharmacy. The review was wide-ranging, covering relevant empirical, theoretical and methodological work, as well as UK policy documents on the topic, without date restrictions. It was carried out between February and July 2015 as a precursor to a research proposal being developed to design, implement and evaluate a complex intervention based in primary care in the NHS for optimising medicines use by patients who take multiple medications (Reeve et al., 2015).

We did not attempt a systematic review of the literature on patients' experiences of polypharmacy with or without multimorbidity at this stage in our preparatory work because we were aware that such evidence was hard to locate (and a sub-project in itself). For example, studies on this topic tend to use different terminology; relevant findings are also often reported in the context of wider studies of patients' experiences of chronic illness using qualitative or mixed methods. For these reasons, relevant evidence is not easily found using mechanical search procedures. Instead, we did three things. We started with the literature that we knew on patient experiences of using medicines and followed up relevant references. Then we began compiling a list of terms that we might use to search the literature more systematically. Finally, we used some of these terms to search Google, PubMed and other targeted sources (such as specific journals) for relevant papers, while continuing to compile our vocabulary of terms for a future more comprehensive review.

This process was very revealing, highlighting major issues with the array of terms used (and variously defined) in research on polypharmacy and medicines usage. A list of the terms we have compiled to date is available (see Supplementary Appendix). For the purposes of this paper, we refer to a few of the 34 studies we identified to illustrate key findings and gaps in this literature. We hope to carry out a more thorough and complete narrative review of this literature using the terms we have identified as part of our ongoing work.

In our initial reading of the policy documents, we noted some variation in how central patients were in them, as well as how limited reference was to relevant research on patient experiences of using medicines. We carried out the present documentary analysis to examine in more depth how patient perspectives had been incorporated and to consider how future research might better inform policy on medicines optimisation.

In the paper, we focus on the current policy advice produced for healthcare professionals in England. This is because, as we describe below, it differs from that of Scotland in promoting person-centred care values and in positing medicines optimisation as a way of managing the rise of polypharmacy and some of the problems associated with it. Our discussion of the implications of our findings is, however, relevant to researchers and policymakers across the UK and in other countries where the rise of polypharmacy is a matter of concern.

\section{Overview of medicines optimisation guidelines in England}

'Medicines optimisation' is a relatively new model of prescribing that is currently being promoted by the NICE and by independent organisations to succeed the established 'medicines management' approach in England. In this section, we examine how the new model is conceptualised and differentiated from the old one in major policy reviews and guidelines as being more centred around patients and less concerned with processes and systems of prescribing.

Primary Health Care Research \& Development 2017; 18: 24-34 
In 2013, the RPS published Medicines optimisation: helping patients to make the most of medicines (Picton and Wright, 2013). It states that

Medicines optimisation is about ensuring that the right patients get the right choice of medicine, at the right time. By focusing on patients and their experiences, the goal is to help patients to: improve their outcomes; take their medicines correctly; avoid taking unnecessary medicines; reduce wastage of medicines; and improve medicines safety. Ultimately medicines optimisation can help encourage patients to take ownership of their treatment.

(Picton and Wright, 2013: introduction).
Developed with input from healthcare professionals, patients and the pharmaceutical industry, the report provides guidance on good practice for healthcare professionals in England, based around four key principles: (1) aim to understand the patient's experience; (2) evidence-based choice of medicines; (3) ensure medicines use is as safe as possible; and (4) make medicines optimisation part of routine practice. The outcomes that these principles are intended to influence are shown in Table 1.

The RPS principles have been agreed by NHS England and used to inform the ongoing development of its Medicines Optimisation Prototype Dashboard (www.england.nhs.uk/ourwork/pe/modash) and wider Medicines Optimisation strategy. They have also been agreed by The Association of

Table 1 Royal Pharmaceutical Society's four guiding principles of medicines optimisation and their intended outcomes

Principle 1: aim to understand Principle 2: evidence-based the patient's experience
Principle 3: ensure medicines Principle 4: make medicines use is as safe as possible

optimisation part of routine practice

Patients feel able to discuss and review their medicines with anyone involved in their care obtained from choosing a from medicines are reduced medicine using best evidence (eg, following NICE guidance, Patients have more local formularies, etc.) and these outcomes are measured confidence in taking their medicines

Patients feel able to ask

Patients' beliefs and preferences about medicines are understood to enable a shared decision about treatment

Patients are able to take/use their medicines as agreed

Patients feel confident enough to share openly their experiences of taking or not taking medicines, their views about what medicines mean to them, and how medicines impact on their daily life
Treatments of limited clinical value are not used and medicines no longer required are stopped

Decisions about access to medicines are transparent and in accordance with the NHS Constitution healthcare professionals when they have a query or a difficulty with their medicines

Patients remain well and there is a reduction in admissions and readmissions to hospitals related to medicines usage

Patients discuss potential side effects and there is an increase in reporting to the Medicines and Healthcare products Regulatory Agency (MHRA)

Patients take unused medicines to community pharmacies for safe disposal
Patients receive consistent messages about medicines because the healthcare team liaise effectively

It becomes routine practice to signpost patients to further help with their medicines and to local patient support groups

Inter-professional and interagency communication about patients' medicines is improved

Medicines wastage is reduced

The NHS achieves greater value for money invested in medicines

The impact of medicines optimisation is routinely measured

NICE = National Institute for Health and Clinical Excellence; NHS = National Health Service

Source: Picton and Wright (2013)

Primary Health Care Research \& Development 2017; 18: 24-34 
the British Pharmaceutical Industry, The Royal College of Nursing, The Royal College of General Practitioners and The Academy of Medical Royal Colleges (Keele Centre for Medicines Optimisation, 2015: 6).

In the same year, The King's Fund, an independent 'think tank', issued Polypharmacy and medicine optimisation: making it safe and sound (Duerden et al., 2013). The authors examine the nature and extent of the problem of polypharmacy in the UK, and consider the implications for policy and practice. A key distinction is made between 'appropriate' and 'problematic' polypharmacy. The former is achieved when: 'prescribing for an individual for complex conditions or for multiple conditions in circumstances where medicines use has been optimised and where the medicines are prescribed according to best evidence', whereas the latter occurs when 'multiple medications are prescribed inappropriately, or where the intended benefit of the medication is not realised' (Duerden et al., 2013: 1).

According to the report, the 'overall intent for the combination of medicines prescribed should be to maintain good quality of life, improve longevity and minimise harm from drugs' (Duerden et al., 2013: 1). So medicines optimisation is intended to support the achievement of appropriate polypharmacy for these various needs and minimise the occurrence of problematic polypharmacy. However, the latter occurs for a number of reasons, such as when the treatments are not evidence based, or the risk of harm is likely to outweigh benefit, or the combination of drugs is hazardous because of interactions between them, or the demands of medicine-taking are unacceptable to patients, or the demands make it difficult to achieve clinically useful medication adherence, or when medicines are prescribed to treat the side effects of other medicines even though other solutions are available to reduce the number of medicines prescribed (Duerden et al., 2013: 1).

The authors go on to propose some solutions to problematic forms of polypharmacy based on processes associated with medicines management and the newer notion of 'medicines optimisation'. They claim that the latter model, with its wider focus on how medicines are or are not used by people, is fundamental to addressing problematic polypharmacy. As they put it: 'Medicines optimisation, or robust medicines management, helps to ensure more appropriate polypharmacy so that the various trade-offs of harm, benefit and patient acceptability and choice have been considered and an explicit decision on the drug to use has been made with the patient' (Duerden et al., 2013: 2). Throughout the report, they stress the need for clinicians to involve patients in decisions on drug use.

In March 2015, following a public consultation from 2013 to 2014, NICE published the guideline NG5: Medicines optimisation: the safe and effective use of medicines to enable the best possible outcomes (NICE, 2015a). The NICE guideline adopts The King's Fund's definitions of appropriate and problematic polypharmacy and describes medicines optimisation as 'a person-centred approach to the safe and effective use of medicines' (NICE, 2015a: 5). It notes that this approach replaces the previous focus on systems, processes and behaviours that was characteristic of medicines management, although the latter is still viewed as 'an important enabler of medicines optimisation' (NICE, 2015a: 8). The eight topic areas covered by the NICE recommendations for practice, and the four for research, are listed in Table 2.

Elsewhere in the UK, NHS Scotland's Polypharmacy Guidance, originally published in 2012, was recently updated in 2015 (Scottish Government

Table 2 National Institute for Health and Social Care Excellence recommendations for practice and research on medicines optimisation: topic areas

Practice recommendations
Systems for identifying, reporting and learning from
medicines-related patient safety incidents
Medicines-related communication systems when
patients move from one care setting to another
Medicines reconciliation
Medication review
Self-management plans
Patient decision aids used in consultations involving
medicines
Clinical decision support
Medicines-related models of organisational and
cross-sector working
Research recommendations
Medication review in children - suboptimal use of
medicines and medicines-related patient safety incidents
Medication review - suboptimal use of medicines and
patient-reported outcomes
Clinical decision support systems
Cross-organisational working

Source: National Institute for Health and Clinical Excellence (NICE) (2015a).

Primary Health Care Research \& Development 2017; 18: 24-34 
Model of Care Polypharmacy Working Group, 2012; 2015; Wilson et al., 2015). Although published over a similar timescale, these guidelines differ in some important respects from those for NHS England. First, they define the positive and negative forms of polypharmacy slightly differently, preferring the terms 'appropriate' and 'inappropriate' polypharmacy, and describing the particular conditions when each is present (Scottish Government Model of Care Polypharmacy Working Group, 2015: 5; Wilson et al., 2015: 109). Second, they do not promote medicines optimisation as a model for dealing with inappropriate forms of polypharmacy. Instead, the Scottish report outlines a '7-step' Medication Review Process to inform patients' and clinicians' decision making about medicines. Finally, this process is not described as a person-centred (or patient-centred) approach as such.

In the rest of the paper we focus on how, unlike Scotland, policy in England has promoted a shift to the more person-centred medicines optimisation model as a strategic response to the rise of polypharmacy. Although the RPS, The King's Fund and NICE guideline all support this strategy, as we show below, their reports vary in how integral patient perspectives and person-centred care values are to them, and also in the extent to which they draw on evidence from existing research on people's experiences of medicines use in general, and polypharmacy in particular.

\section{Centrality of patient perspectives and person-centred care in the guidelines}

In the RPS report, medicines optimisation is represented as a 'step change' from the medicines management approach, focussing on patients and the outcomes that matter to them, rather than on professionals and the systems and processes that shape how medicines are used (Picton and Wright, 2013: introduction). While the RPS report emphasises the divergence between the two models, both The King's Fund report and NICE guideline highlight the convergence: they tend to represent medicines optimisation as a broad approach that is facilitated by processes and systems of medicines management.

This difference in perception of the two models is reflected in the extent to which the respective guidelines are formulated around patients. In the RPS guidelines, the need to understand patient experiences is relatively strongly represented, forming one of its four fundamental principles of medicines optimisation. Many of the RPS recommendations are also written from the patient's viewpoint (see Table 1).

In The King's Fund report, the importance of determining patient perspectives is a running theme, although here it is represented more as a matter for clinicians to address on an individual basis rather than, say, as a guiding principle of care or a systemic issue, and there are no references to practical information on how to go about this. There is just a short section - less than a page in the 56-page report - summarising what is known about patient experiences of polypharmacy.

Similarly, the 47-page NICE guideline has a one-page section describing 'person-centred care' (which it treats as equivalent to 'patient-centred care') that is somewhat separate from, and not integrated with, the rest of the report. Moreover, the actual recommendations are mainly concerned with processes and systems for making safe and cost-effective decisions with less space given to ways of facilitating the identification and achievement of person-centred goals. This is also reflected in the companion information NICE produced for the public (NICE, 2015b).

Despite their common support for medicine optimisation, a model that is conceptually distinguished from medicines management by its greater focus on patient perspectives, only The King's Fund report engages with real examples of the sorts of issues patients have experienced, albeit briefly. In a short section on 'Polypharmacy and the patient experience', the authors describe some of the evidence about people's strategic use of medicines and difficulties coping with the demands of their drug regimens (Duerden et al., 2013: 32). They also discuss a key issue that is glossed over in the other reports - that patients and professionals do not always agree about medicines usage - and suggest ways of dealing with this. As they point out, 'Patients may not want to take multiple medicines, or prefer one treatment over another. Advice should be given on which interventions may be most likely to minimise side effects, reduce symptoms and improve outcomes. Regimens may need to be tailored to fit with patient preferences and "compromise" may be required" (Duerden et al., 2013: 1-2). 
Expanding on these ideas, the authors go on to add that

Compromises may often need to be reached between the view of the prescriber in delivering interventions intended to improve outcome, and the choice made by the patient, based on the demands of the medication regimen. The alternative is the potentially wasteful process of prescribing where the patient does not take the medicines appropriately, or does not take them at all, but the prescriber unwittingly continues to supply prescriptions. Various estimates of long-term drug use indicate that as many as 40 per cent of people on long-term prescriptions do not take them as intended [ref].

(Duerden et al., 2013: 32).

Closely related to this theme, which again only The King's Fund report mentions, is the notion of the 'demands' of the drug regimen and the 'pill burden' that patients often find unacceptable, which are two of the manifold reasons why patients may sometimes choose not to use medicines (Duerden et al., 2013: 1).

\section{Utilisation of research in the guidelines}

The three reports were each informed by associated reviews of the literature. However, there was noticeable variation in the extent to which they covered qualitative studies of people's use of medicines. For example, the RPS report draws on an undated review of evidence (RPS, nd) that it published separately online at www.rpharms.com/ medicines-safety/medicines-optimisation.asp. Most of the 16 references in the report itself refer to research on prescribing practices. The consultation document (NICE, 2014) that the NICE guideline was developed from summarises evidence from systematic reviews, randomised controlled trials and observational studies but not qualitative studies of people's experiences of using medicines. Only The King's Fund report considers some of the evidence from qualitative studies of people's experiences of using medicines in its more comprehensive review.

The under-utilisation of qualitative research on people's experiences of using medicines is disappointing given that there is a large international literature on the topic and on patients' self-management of chronic conditions, which often includes a focus on medicines usage. This work has examined, among other things, the reasons why some people do not always take their medicines as prescribed (eg, Britten, 2007), the disruptive effects of being ill and fitting complex care regimens into everyday life, and the various strategies that people have adopted to minimise these effects (Demain et al., 2015). However, while information on these and other aspects of medicines usage is (and was) already available in the literature and could have been better utilised in the guidelines, this evidence base is not without limitations.

Much of what we know about people's use of medicines is based on evidence from studies of patients with single conditions, such as diabetes or asthma. Only a small proportion of the existing literature focusses on people who take multiple medications for multiple conditions. This is important because, while people may take two or more medicines for a single condition, those taking multiple medicines for multiple conditions, and those prescribing for them, face some additional issues. Although it is not our intention to fully review the literature on people's experiences of polypharmacy and multimorbidity here, we highlight below some of the complex issues that have been identified by studies carried out in the UK to date, which are relevant to policy and guidelines on medicines optimisation but not so far addressed by them.

In an early study of the views of people with multimorbidity on their complex drug regimens, Townsend et al. (2003; 2006) found tensions in their experiences of 'regular' versus 'flexible' regimens for different drugs. They also found that people preferred to minimise their use of prescribed medicines and maximise their use of alternative ways of managing their conditions. Another study of people with type 2 diabetes and cardiovascular diseases found that they believed healthcare professionals were more likely to overprescribe for those with comorbid conditions, and that more people prioritised their medicines prescribed for their diabetes than for their cardiovascular disease (Stack et al., 2008). Lindsay (2009) found that people with multiple chronic conditions often prioritised one main condition because it was unpredictable, or it was not controlled through tablets, or it tended to set off other problems.

Primary Health Care Research \& Development 2017; 18: 24-34 
Some studies have also begun to document the strategies that people have developed to deal with the issues of using multiple medications. For example, a qualitative longitudinal study of people's experiences of multimorbidity, which examined people's shifting priorities over time, found that the prescription of medicines from different sources and with various instructions led to some confusion, but also to the development of pragmatic routines enabling people to take control (Morris et al., 2011). The authors further observed that: 'Medication management emerged as an anchor or point around which multiple condition management could be changed (or abandoned) and represented a point whereby a person could either take or abandon control' (Morris et al., 2011: 158). Another study of patients who were regularly prescribed four or more medicines for chronic conditions found that while some did adapt to their long-term medicines use others did so at a cost to their quality of life (Krska et al., 2013).

We believe that the lack of research on these and other issues experienced by people taking multiple medicines for single or multiple conditions in the UK has limited the size and quality of the evidence-base available to potential users. As a result, policymakers have lacked relevant evidence to draw upon in preparing guidelines on polypharmacy, medicines optimisation and multimorbidity; clinicians have lacked meaningful and practical information on how to practice optimal prescribing for people with complex or multiple conditions; and patients have lacked insights from other patients with first-hand experience about how they themselves have developed strategies for self-optimising medications, and whether these have been successful or not from their point of view. In the last part of the paper, we consider some of the ways in which researchers might work to improve the evidence base to inform the future production of person-centred guidelines on medicines optimisation.

\section{Implications for research}

Below we suggest five ways in which researchers could help policymakers to both make better use of available knowledge on the topic and provide them with more evidence about patient experiences of

Primary Health Care Research \& Development 2017; 18: 24-34 polypharmacy, to inform the future development of guidelines on medicines optimisation and ensure that they are truly person-centred.

\section{Facilitate reviews of research}

One possible reason why the current policy reviews and guidelines make partial and limited use of the available research on medicines use is because of the amorphous nature of the literature. The literature on patient experiences of using multiple medicines is difficult to identify and review because of a lack of consistency and clarity in the terminology that is used. There is, for example, no commonly accepted definition of the number of medicines involved in polypharmacy. In a review of polypharmacy terminology, Bushardt et al. (2008: 386) found that six or more drugs was the most commonly used number. In a study of polypharmacy among people with stroke and other morbidities, Gallacher et al. (2014) observed that five or ten tend to be the most commonly used thresholds. Other numbers have also been used to indicate progressive levels of polypharmacy. Definitions may be limited to prescription drugs or include over the counter drugs as well.

Similarly, while there is general acceptance that the individualisation of drug treatments is a good thing, there is no common understanding of what this means. As Denford et al. (2014) have shown, the terms 'personalised' and 'individualised' care have been variously and sometimes vaguely defined in published studies. As previously noted, the terms 'patient-centred' and 'person-centred' care are sometimes used interchangeably (as in the NICE guideline) or the latter can be used to refer to a philosophy of care that is distinct from that of the former (eg, Ekman et al., 2011; Rasmussen et al., 2014). Given the proliferation of terms in this field of research, it is important that researchers carefully select and clearly define the terms used in studies, and differentiate them from alternatives where necessary, to help facilitate the identification and review of relevant work by users of research knowledge.

\section{Improve the evidence base on patient experiences of polypharmacy and multimorbidity}

As we have noted, relatively little is known about patient experiences of polypharmacy, 
particularly those with multimorbidity. There is a particular need for more research on the following topics:

- patient experiences of polypharmacy, especially those taking multiple medicines for multiple chronic conditions, including the disruptiveness and burden of their drug regimens, and the making and balancing of different priorities;

- patients' and prescribers' understanding of and attitudes to medicines optimisation, including their views on the nature and acceptability of compromises;

- patient and prescriber perspectives on the acceptability, safety and effectiveness of particular interventions designed to optimise medicines use;

- patient and prescriber perspectives on the barriers and facilitators to the optimisation of medicines prescribed by different practitioners across primary and secondary care settings.

These topics will in some cases require a longitudinal approach, for example, examining how patients' priorities and use of medicines shift over time, what influences this and how adaptations to drug regimens can be made in response. They also require modes of analysis that bring out the ways in which the design and operation of systems of care are reflected and embodied in individual patient's experiences. This includes sensitivity to the language used by patients and carers to describe what they regard as good quality care that meets their needs and priorities, which is not always the same as that used by services or in policy. An article on patients' and carers' experiences of obtaining repeat prescriptions provides an example of this approach (Wilson et al., 2013).

\section{Improve the evidence base on medicine optimisation interventions}

Although the policy documents support a move to medicines optimisation in England, they contain little information and practical guidance for healthcare professionals and patients on how to work together to achieve this in practice. Skinner (2015) has also revealed a lack of clinical protocols for polypharmacy that are specific to primary care, although she acknowledges the difficulties of providing guidelines for professionals treating individual patients with multiple conditions, each of whom have 'unique' health priorities (Skinner, 2015: 4-5).
In the proposal that we are developing on the back of this work, we are planning to design and evaluate a generalist approach to prescribing that can be tailored to individuals and their shifting circumstances and priorities (Reeve et al., 2015). However, this is just one possible study of one approach and others are required to investigate the nature and challenges, and benefits and costs, of interventions for optimising medicines in different contexts. A recent study in Ireland of how general practitioners make compromises when prescribing for patients with multimorbidity is a helpful contribution to knowledge in this regard (Sinnott et al., 2015).

\section{Better use of existing and emerging theories, concepts and tools}

There are a number of theoretical frameworks that could be used to inform future conceptual and empirical research on polypharmacy and medicines optimisation. These include debates around the notions of 'patient-centred' and 'person-centred' care and how these fit with ideas about medicines optimisation and evidence-based medicine. They also include theories such as generalism and how this might provide the basis for reshaping practice around people rather than their diseases (Reeve and Bancroft, 2014). There are also concepts such as 'burden of treatment' (May et al., 2014) and 'minimally disruptive medicine' that are salient and informing the development of tools for measuring the impact of interventions in terms that are more relevant to patients (Lepping, Montori and Gionfriddo, 2015).

These theoretical approaches, in their different ways, provide possible alternatives to existing conceptual models of prescribing, which have been largely influenced by ideas about patients' adherence (and previously compliance) to medication regimens. For a long time, these ideas have failed to fundamentally engage with patients' perspectives on how they routinely use medicines to better enable them to live their lives and so a new approach is needed.

\section{Improved patient and public involvement in research and in the development of guidelines}

One way of improving the centrality of patients in guidelines purporting to promote patient- or personcentred care is to increase their meaningful Primary Health Care Research \& Development 2017; 18: 24-34 
involvement in research and in the development of guidelines and clinical protocols. It is encouraging to see that NHS England has so far carried out two patient engagement workshops in the course of developing their Medicines Optimisation strategy (www.england.nhs.uk/ourwork/pe/mo-dash). Krahn and Naglie (2008) have argued that patients' perspectives, experiences and choices should be considered at every stage of the development and implementation of clinical practice guidelines. They suggest five ways of doing this: finding preferencerelated evidence; integrating preference-related evidence into recommendations; using guidelines in individual decision making; including patients in the guideline development process; and evaluating guidelines with preferences in mind (Krahn and Naglie, 2008: 437).

More recently, Montori et al. (2013), working out of the Knowledge and Research Unit at the Mayo Clinic in the United States, have similarly called for the incorporation of patient preferences into practice guidelines. They suggest that guideline panels should include frontline patients and clinicians; consult with clinical and methodological experts and seek testimony from individuals who are experts in patient preferences, patients and caregivers', as well as commission relevant reports; and rely on patient input to 'drive consideration of the full range of outcomes patients experience and consider critical in deciding what to do' (Montori et al., 2013: 2504). They also suggest that panels should refrain from making 'strong' recommendations when 'the best course of action heavily depends on the patient's context, goals, values, and preferences'; instead they advise panels to make 'conditional recommendations' that reflect this scenario and presents options in a way that facilitates shared decision making (Montori et al., 2013: 2504).

In what we regard as a refreshing dose of realism, they conclude by arguing that

Panels should become much more comfortable with ambiguity, both in the tradeoffs involved and in the recommendations given, and explicitly report how patient preferences and context were considered in formulating the panels' recommendations. Clinicians need guidance and clear guidance helps and supports efficient practices. Yet, panels must be wise in recognizing when this expediency is appropriate for patient care and when it

Primary Health Care Research \& Development 2017; 18: 24-34 hinders patient-centred care. Clinicians should remember that taking care of patients is supposed to be difficult. Although guidelines may simplify this task, when patient preferences and context matter, guidelines must not replace clinicians' compassionate and mindful engagement of the patient in making decisions together. This is the optimal practice of evidence-based medicine.

(Montori et al., 2013: 2504).

We would reiterate that patients, too, need to be involved as members of panels and, more generally, in discussions around 'ambiguity' and 'compromises' in prescribing and debates about the ethics and values which inform decision making in prescribing.

\section{Conclusion}

In this paper, we have drawn attention to the different ways in which patient perspectives and person-centred care values have been represented in official and influential independent policy documents on medicines optimisation in England. Whereas, understanding patient perspectives is a fundamental principle of the RPS guidelines, the more recent NICE guideline focusses mainly on the safe and effective prescribing of medicines, and less on the identification and achievement of personal goals, which are also important to patients. We have argued that this partly reflects a lack of utilisation of existing qualitative research on patient experiences of polypharmacy, especially where linked to multimorbidity, as well as a lack of basic research specifically on this topic. We have suggested some topics that we believe are priorities for further research, and highlighted ways in which patients can be more fully involved in the process of developing guidelines. We hope that researchers, funders of research and policymakers will use our suggestions to help improve the construction of guidelines on medicines optimisation that are meant to enable patients to get the most from their medicines.

\section{Acknowledgements}

We would like to acknowledge the support of other members of the sub-team involved in 
developing the Programme Grant linked to this work: patient and public representatives Jim Harris and Ed Ranson, and Professor Richard Byng.

\section{Financial Support}

This work was supported by the National Institute for Health Research (NIHR) Collaboration for Leadership in Applied Health Research and Care South West Peninsula at the Royal Devon and Exeter NHS Foundation Trust. The views expressed are those of the authors and not necessarily those of the NHS, the NIHR or the Department of Health.

\section{Conflicts of Interest}

None.

\section{Supplementary material}

To view supplementary material for this article, please visit http://dx.doi.org/10.1017/S14634236 16000207

\section{References}

Britten, N. 2007: Understanding medicine taking in context. In J. Dowell, B. Williams and D. Snadden, editor. Patientcentered prescribing: seeking concordance in practice. Oxford: Radcliffe Publishing, 29-43.

Bushardt, R.L., Massey, E.B., Simpson, T.W., Ariail, J.C. and Simpson, K.N. 2008: Polypharmacy: misleading, but manageable. Clinical Interventions in Aging 3, 383-89.

Demain, S., Gonçalves, A.-C., Areia, C., Oliveira, R., Marcos, J., Marques, A., Parmar, R. and Hunt, K. 2015: Living with, managing and minimising treatment burden in long term conditions: a systematic review of qualitative research. PLOS One 10, 18pp.

Denford, S., Frost, J., Dieppe, P., Cooper, C. and Britten, N. 2014: Individualisation of drug treatments for patients with long-term conditions: a review of concepts. BMJ Open 4, e004172. 11pp. doi: 10.1136/bmjopen-2013-004172.

Duerden, M., Avery, T. and Payne, R. 2013: Polypharmacy and medicines optimisation: making it safe and sound. London: King's Fund. 56pp.

Ekman, I., Swedberg, K., Taft, C., Lindseth, A., Norberg, A., Brink, E., Carlsson, J., Dahlin-Ivanoff, S., Johansson, I.-L., Kjellgren, K., Lidén, E., Öhlén, J., Olssen, L.-E., Rosén, H., Rydmark, M. and Sunnerhagen, K.S. 2011: Person-centred care - ready for prime time. European Journal of Cardiovascular Nursing 10, 248-51.
Gallacher, K.I., Batty, G.D., McLean, G., Mercer, S.W., Guthrie, B., May, C.R., Langhorne, P. and Mair, F.S. 2014: Stroke, multimorbidity and polypharmacy in a nationally representative sample of 1,424,378 patients in Scotland: implications for treatment burden. BMC Medicine 12, 151. doi:10.1186/s12916-014-0151-0.

Keele Centre for Medicines Optimisation 2015: NHS England Medicines Optimisation Prototype Dashboard Evaluation report. Keele Centre for Medicines Optimisation, Keele University, Keele.

Krahn, M. and Naglie, G. 2008: The next step in guideline development: incorporating patient preferences. JAMA 300, 436-38.

Krska, J., Morecroft, C.W., Poole, H. and Rowe, P.H. 2013: Issues potentially affecting quality of life arising from long-term medicines use: a qualitative study. International Journal of Clinical Pharmacology 35, 1161-169.

Leppin, A.L., Montori, V.M. and Gionfriddo, M.R. 2015: Minimally disruptive medicine: a pragmatically comprehensive model for delivering care to patients with multiple chronic conditions. Healthcare 3, 50-63.

Lindsay, S. 2009: Prioritizing illness: lessons in self-managing multiple chronic diseases. Canadian Journal of Sociology 34 , 983-1002.

May, C.R., Eton, D.T., Boehmer, K., Gallacher, K., Hunt, K., MacDonald, S., Mair, F.S., May, C.M., Montori, V.M., Richardson, A., Rogers, A.E. and Shippee, N. 2014: Rethinking the patient: using burden of treatment theory to understand the changing dynamics of illness. BMC Health Services Research 14, 281.

Montori, V.M., Brito, J.P. and Murad, M.H. 2013: The optimal practice of evidence-based medicine: incorporating patient preferences in practice guidelines. JAMA 310, 2503-504.

Morris, R.L, Sanders, C., Kennedy, A.P. and Rogers, A. 2011: Shifting priorities in multimorbidity: a longitudinal study of patient's prioritization of multiple conditions. Chronic Illness 7, 147-61.

National Institute for Health and Clinical Excellence (NICE) 2014: Medicines optimisation: the safe and effective use of medicines to enable the best possible outcomes. Clinical Guideline. Methods, Evidence and Recommendations. Draft for Consultation. London: National Institute for Health and Clinical Excellence.

National Institute for Health and Clinical Excellence (NICE) 2015a: Medicines optimisation: the safe and effective use of medicines to enable the best possible outcomes. NICE guideline (NG5). London: National Institute for Health and Clinical Excellence. Retrieved 7 June 2016 from Nice.org.uk/ guidance/ng5.

National Institute for Health and Clinical Excellence (NICE) 2015b: The safe and effective use of medicines (medicines optimisation). Information for the Public. Retrieved 14 October 2015 from www.nice.org.uk/guidance/ng5/ informationforpublic.

Picton, C. and Wright, H. 2013: Medicines optimisation: helping patients to make the most of medicines. Good practice

Primary Health Care Research \& Development 2017; 18: 24-34 


\section{Janet Heaton et al.}

guidance for healthcare professionals in England. London: Royal Pharmaceutical Society. 12pp.

Rasmussen, E., Jørgensen, K. and Leyshon, S. 2014: Personcentred care - co-creating a healthcare sector for the future. Esbjerg: DNV GL and Monday Morning/Sustainia, Rosendahls. 202pp.

Reeve, J. and Bancroft, R. 2014: Generalist solutions to overprescribing: a joint challenge for clinical and academic primary care. Primary Health Care Research and Development $15,72-79$.

Reeve, J., Dickenson, M., Harris, J., Ranson, E., Dohnhammer, U., Cooper, L., Krska, J., Byng, R. and Britten, N. 2015: Solutions to problematic polypharmacy: learning from the expertise of patients. British Journal of General Practice, doi:10.3399/bjgp15X685465.

Royal Pharmaceutical Society (RPS) (nd) Medicines optimisation: the evidence in practice. Retrieved 14 October 2015 from www.rpharms.com/promoting-pharmacy-pdfs/moevidence-in-practice.pdf.

Scottish Government Model of Care Polypharmacy Working Group 2012: Polypharmacy guidance. Edinburgh: Quality and Efficiency Support team \& Scottish Government Health and Social Care Directorates, NHS Scotland.

Scottish Government Model of Care Polypharmacy Working Group 2015: Polypharmacy Guidance, second edition. Edinburgh: Scottish Government.
Sinnott, C., Mc Hugh, S., Boyce, M.B. and Bradley, C. P. 2015: What to give the patient who has everything? A qualitative study of prescribing for multimorbidity in primary care. British Journal of General Practice 65, 8pp.

Skinner, M. 2015: A literature review: polypharmacy protocol for primary care. Geriatric Nursing 36, 5pp.

Stack, R.J., Elliot, R.A., Noyce, P.R. and Bundy, C. 2008: A qualitative exploration of multiple medicines beliefs in co-morbid diabetes and cardiovascular disease. Diabetic Medicine 25, 7pp. doi: 10.1111/j.1464-5491.2008.02561.x.

Townsend, A., Hunt, K. and Wyke, S. 2003: Managing multiple morbidity in mid-life: A qualitative study of attitudes to drug use. British Medical Journal 327(837), 6pp.

Townsend, A., Wyke, S. and Hunt, K. 2006: Self-managing and managing self: Practical and moral dilemmas in accounts of living with chronic illness. Chronic Illness, 2, 185-94.

Wilson, M., Mair, A., Dreischulte, T. and Witham, M.D. 2015: Prescribing to fit the needs of older people- the NHS Scotland Polypharmacy Guidance, 2nd edition. Journal Royal College Physicians 45, 108-13.

Wilson, P.M., Kataria, N. and McNeilly, E. 2013: Patient and carer experience of obtaining regular prescribed medication for chronic disease in the English National Health Service: a qualitative study. BMC Health Services Research 13, 192. 11pp. doi: 10.1186/1472-6963-13-192. 